\title{
Fully Developed Triplet Proximity Effect
}

\author{
V. Braude and Yu. V. Nazarov \\ Kavli Institute of Nanoscience, Delft University of Technology, 2628 CJ Delft, The Netherlands
}

(Received 2 October 2006; published 15 February 2007)

\begin{abstract}
We present a model for the fully developed proximity effect in superconductor-ferromagnet heterostructures. Within the circuit-theory approximation, we evaluate the Green functions, the density of states, and the Josephson current which depend essentially on the magnetic configuration.
\end{abstract}

The research of heterostructures that combine superconducting (S) and ferromagnetic (F) elements gives insight into the problem of the mutual influence of superconductivity and ferromagnetism, allows realization of exotic $\mathrm{S}$ states such as the Larkin-Ovchinnikov-Fulde-Ferrell state [1] and triplet ordering [2], and promises applications that utilize the spin degree of freedom [3]. While this research started more than three decades ago [4], interest in the above topics has produced important recent developments, both theoretical and experimental. Those concern Josephson $\pi$ junctions [5,6], triplet superconductivity $[2,6,7]$, and Josephson spin valves $[8,9]$.

We refer to the proximity effect that takes place in nonmagnetic dirty superconducting/normal $(\mathrm{S} / \mathrm{N})$ structures as "conventional." In the normal part of a $\mathrm{S} / \mathrm{N}$ structure, $\mathrm{S}$ correlations persist at distances of the order of normal-metal coherence length $\xi_{N}$. This length scale can grow large at sufficiently small temperatures $T$. In a diffusive material, $\xi_{N}=\sqrt{\hbar D / 2 \pi} \overline{k_{B} T}, D$ being the diffusivity. In contrast to this, $S$ correlations in a ferromagnet, where an exchange field $h$ is present, are quenched at a much shorter scale $\xi_{h}=\sqrt{\hbar D / h}$. Hence one might conclude that the proximity and Josephson effects are strongly suppressed in S/F heterostructures. However, some experiments [10] seem to contradict this statement, indicating proximity correlations at a much larger scale. Although these experiments may be explained by interface effects [11], they have motivated a proposal of an interesting mechanism for long-range proximity effect in ferromagnets $[2,6]$. It was shown that inhomogeneity in the direction of exchange field generates $\mathrm{S}$ correlations of two electrons with the same spin, i.e., triplet correlations. Such a triplet proximity effect (TPE) is not suppressed by an exchange field and penetrates the ferromagnet at the scale of $\xi_{N}$. Recently, a substantial Josephson current has been reported for a fully polarized ferromagnet [7]. The experiment can only be explained by TPE.

An immediate problem is that the theoretical predictions so far have been elaborated by assuming that TPE is weak and can be treated perturbatively. This makes it difficult to determine an unambiguous experimental signature of TPE to distinguish it from the conventional effect. Experimentally, the Josephson current due to TPE [7] does not seem to be smaller than that due to a fully developed conventional proximity effect.

In this work, we address a fully developed TPE, that is, the TPE that significantly changes the density of states (DOS) near the Fermi energy. We show that the DOS increases. This is in contrast with complete suppression of DOS by a fully developed conventional proximity effect. Similar to the conventional effect, the change in the DOS is restricted to the energy window $\simeq E_{\mathrm{Th}}$, the Thouless energy of the structure, provided $E_{\mathrm{Th}} \ll \Delta, \Delta$ being the energy gap in the superconductor. Therefore the corresponding Josephson current is of the same order as for the conventional proximity effect, though its magnitude essentially depends on the magnetization configuration in the structure. For magnets where both spin directions are present at the Fermi surface (F metals), we find both $\pi$ junctions $[5,6]$ (with negative supercurrents) and common 0 junctions (with positive supercurrents), depending on the magnetization directions. For fully polarized magnetic materials (half-metals), we find a continuous dependence of the equilibrium $\mathrm{S}$ phase difference on magnetization directions. Finite current may be induced thereby at fixed zero phase difference.

We concentrate on a $S / F / S$ heterostructure fabricated by deposition of two $\mathrm{S}$ electrodes onto a conducting $\mathrm{F}$ film [Fig. 1(a)] [7]. We proceed with the so-called circuit theory [12] that is a finite-element technique for the semiclassical Green function method [13], which has been applied to S/F structures in Ref. [9]. Circuit theory is convenient since we aim at presenting an idealized TPE without consideration and subsequent optimization of concrete geometry of the structure. Besides, it allows simple analytical presentation of the results.

We build a minimal circuit-theory model for a $\mathrm{F}$ metal, later adjusting it to a half-metal. The left and right parts of the structure contain regions where $\mathrm{S}$ and magnetic correlations meet [points 1 and 3 in Fig. 1(a)]. Following [9], we represent each region by a normal-metal node connected to a $\mathrm{S}$ reservoir and a ferromagnetic insulating reservoir (FIR). The role of FIR is to represent the exchange field $h_{k}(k=1,2,3)$ induced in the node. The middle of the structure (point 2) is represented as node 2 connected to nodes 1 and 3, and another FIR. To enable TPE, we allow arbitrary magnetization directions of all FIRs. It is assumed 
a)

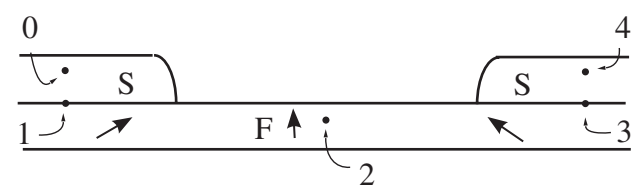

b)

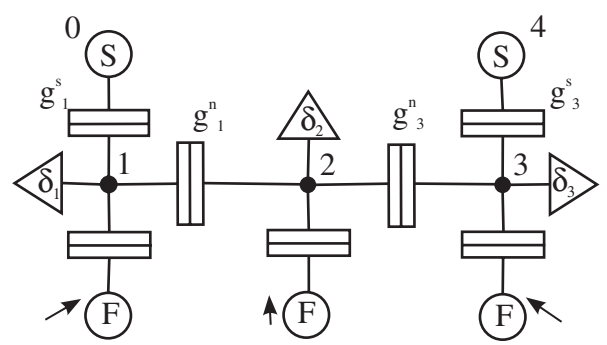

c)

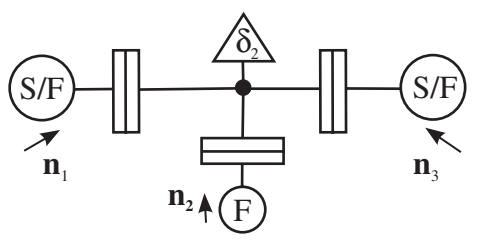

FIG. 1. Building up the circuit-theory model. (a) S/F/S structure exhibiting TPE [7]. (b) The corresponding circuit consists of three nodes representing parts $1-3$ of the structure. (c) Nodes 1 and 3 are replaced by effective S/F reservoirs.

for simplicity that all connectors are of tunnel nature. The finite volume of each node and related decoherence between electrons and holes is taken into account by introducing "leakage" matrix currents [12] inversely proportional to the mean level spacing $\delta_{i}$ in each node. This defines the circuit presented in Fig. 1(b).

The relevant variables are the (retarded) Green functions $G_{1,2,3}$ at the nodes. These Green functions are energydependent $4 \times 4$ matrices in the Nambu and spin space. They are determined from the equations that express conservation of the "matrix currents" at each node. These equations read $(k=1,3)$

$$
\begin{array}{r}
{\left[i \frac{G_{Q}}{\delta_{k}} h_{k}\left(\boldsymbol{n}_{k} \cdot \boldsymbol{\sigma}\right) \tau_{3}+\frac{g_{k}^{s}}{2} G_{k}^{s}-i \frac{G_{Q}}{\delta_{k}} \boldsymbol{\epsilon} \tau_{3}+\frac{g_{k}^{n}}{2} G_{2}, G_{k}\right]=0,} \\
{\left[i \frac{G_{Q}}{\delta_{2}} h_{2}\left(\boldsymbol{n}_{2} \cdot \boldsymbol{\sigma}\right) \tau_{3}-i \frac{G_{Q}}{\delta_{2}} \boldsymbol{\epsilon} \tau_{3}+\sum_{l=1,3} \frac{g_{l}^{n}}{2} G_{l}, G_{2}\right]=0 .}
\end{array}
$$

Here, $G_{Q} \equiv e^{2} / \pi \hbar$ is the conductance quantum and $\boldsymbol{\tau}$ and $\boldsymbol{\sigma}$ are vectors composed of Pauli matrices in the Nambu and spin space, respectively. The first term in these equations is the matrix current into the corresponding FIRs. The magnetization direction in FIR $k$ is given by a unit vector $\boldsymbol{n}_{k}$. $G_{k}^{s}$ is a Green function of the $\mathrm{S}$ reservoir $k$. We assume for simplicity that the relevant energies are much smaller than the $\mathrm{S}$ gap in the reservoirs, so we can use the energyindependent $G_{k}^{s}=\tau_{1} \cos \varphi_{k}+\tau_{2} \sin \varphi_{k}, \varphi_{k}$ being the $\mathrm{S}$ phase. Each $\mathrm{S}$ reservoir $k$ is connected to node $k$ by a tunnel junction with conductance $g_{k}^{s}$. It would induce a proximity effect mini-gap $\Delta_{k}=g_{k}^{s} \delta_{k} / 2 G_{Q}$ in node $k$ if no other connections were present. The parameters $g_{1,3}^{n}$ give the conductances of connections between the normal nodes.

The two energy scales of the model are the typical exchange field $h$ and the Thouless energy $E_{\mathrm{Th}} \simeq$ $g^{n} \delta / G_{Q}$. The condition $h \leq E_{\mathrm{Th}}$ corresponds to a short $\mathrm{S} / \mathrm{F} / \mathrm{S}$ structure with dimensions shorter than or of the order of $\xi_{h}$. In this case, the singlet proximity effect overshadows TPE: singlet anomalous components of $G$ in all nodes either exceed or are of the same order as the triplet ones. Since we want to single out the TPE, we turn to the opposite limit $E_{\mathrm{Th}} \ll h$ of longer structures.

The best separation between the islands is achieved in the limit $\delta_{2} \gg \delta_{1,3}$, where regions 1 and 3 adjacent to the superconductors are much longer than the middle part of the system. We will see that in this limit the $\mathrm{S}$ correlations in nodes 1 and 3 are between electrons of opposite spin. As to node 2, the correlations are between electrons of the same spin only: there TPE is present in its purest form.

Under these conditions, the last term in the first expression of Eq. (1) can be neglected and $G_{1,3}$ can be determined separately from $G_{2}$. Owing to their big size, nodes 1 and 3 act as effective reservoirs for node 2 [Fig. 1(c)]. The Green functions at these nodes are determined by the competition between the corresponding $S$ reservoir and FIR and read [9] $\left(k=1,3 ; h_{k}>\Delta_{k} ; \tau_{ \pm}=\left[\tau_{1} \pm i \tau_{2}\right] / 2\right)$ :

$$
G_{k}=\frac{h_{k} \tau_{3}-i\left(\Delta_{k}^{*} \tau_{+}+\Delta_{k} \tau_{-}\right)\left(\boldsymbol{n}_{k} \cdot \boldsymbol{\sigma}\right)}{\sqrt{h_{k}^{2}-\left|\Delta_{k}\right|^{2}}},
$$

where we assume $\epsilon \ll h_{k}, \Delta_{k}$. As seen from the structure of (2), the effective S/F reservoirs supply $S$ correlations that are different for opposite spin directions. These correlations are most pronounced if $h_{k} \simeq \Delta_{k}$. We will see that this condition is optimal for TPE. At this stage of research it is difficult to immediately relate this condition to the specifics of the structures in hand, such as geometry, film thickness, transparency of the S/F interface, etc. Since the observations of [7] suggest that TPE is close to the optimal one, we are convinced that this condition is realizable.

Now node 2 is connected to reservoirs only. Its Green function is determined by the balance of the matrix currents into these reservoirs. The two S/F reservoirs are connected in parallel, so that their net effect is additive and can be represented by a matrix $M=\left(g_{1}^{n} G_{1}+\right.$ $\left.g_{3}^{n} G_{3}\right) /\left(g_{1}^{n}+g_{3}^{n}\right) \equiv M_{0} \tau_{3}-i\left(\boldsymbol{M}^{*} \tau_{+}+\boldsymbol{M} \tau_{-}\right) \cdot \boldsymbol{\sigma}$. It is important that the resulting $G_{2}$ splits into two independent blocks corresponding to two spin projections on $\boldsymbol{n}_{2}$. The separation into blocks allows us to treat half-metals on equal footing with $\mathrm{F}$ metals. While for a $\mathrm{F}$ metal both blocks contribute to physical quantities, only a single block does so in a half-metal. To implement this in circuit theory, the conductances of connectors 1 and 3 to the central node are made spin dependent. The conductance for spin-down electrons is then set to zero. It is implied that in any case the S/F reservoirs support both spin directions [14], otherwise they would not be superconducting. 
We write the block structure as follows:

$$
G_{2}=S\left[\begin{array}{cc}
G_{\downarrow} & 0 \\
0 & G_{\uparrow}
\end{array}\right] S^{-1},
$$

where $S \equiv\left(1-\tau_{3}\right) / 2-i \sigma_{y}\left(\boldsymbol{n}_{2} \cdot \boldsymbol{\sigma}\right)\left(1+\tau_{3}\right) / 2$. In this notation,

$$
G_{\uparrow}=\frac{1}{\sqrt{A_{\uparrow}}}\left[\begin{array}{cc}
M_{0}-i \epsilon / E_{\mathrm{Th}} & -i\left(M_{x}^{*}-i M_{y}^{*}\right) \\
-i\left(M_{x}+i M_{y}\right) & -M_{0}+i \epsilon / E_{\mathrm{Th}}
\end{array}\right],
$$

where $A_{\uparrow} \equiv\left(M_{0}-i \epsilon / E_{\mathrm{Th}}\right)^{2}-\left|M_{x}+i M_{y}\right|^{2}$ and the $z$ axis is chosen in the direction of $\boldsymbol{n}_{2}$. The result for $G_{\downarrow}, A_{\downarrow}$ is obtained by replacing $M_{x} \pm i M_{y}$ with $M_{x} \mp i M_{y}$. The advantage of such notations is that the block structure is made explicit. The nondiagonal elements of $G_{\uparrow}$ correspond to triplet anomalous averages $\left\langle\psi_{\uparrow} \psi_{\uparrow}\right\rangle,\left\langle\psi_{\uparrow}^{\dagger} \psi_{\uparrow}^{\dagger}\right\rangle$, whereas the singlet-pairing averages $\left\langle\psi_{\uparrow} \psi_{\downarrow}\right\rangle,\left\langle\psi_{\uparrow}^{\dagger} \psi_{\downarrow}^{\dagger}\right\rangle$ vanish. This manifests a pure TPE. The fully developed effect is characterized by $h_{k} \geq\left|\Delta_{k}\right|$, in which case the nondiagonal and diagonal elements of $G_{\uparrow, \downarrow}$ are of the same order of magnitude.

The triplet anomalous averages $\left\langle\psi_{\uparrow} \psi_{\uparrow}\right\rangle\left(\left\langle\psi_{\downarrow} \psi_{\downarrow}\right\rangle\right)$ acquire a phase factor $e^{i \chi}\left(e^{-i \chi}\right)$ upon rotation by an angle $\chi$ about the $z$ axis. This leads to an interesting interplay between the $S$ phase difference $\phi_{3}-\phi_{1} \equiv \phi$ and the relative longitude angle between $\boldsymbol{n}_{1}$ and $\boldsymbol{n}_{3}, \chi_{3}-\chi_{1} \equiv \chi$. To see this explicitly, we express the magnetization direction vectors $\boldsymbol{n}_{1,3}$ in the spherical coordinates ( $\chi$ and $\theta$ standing for the longitude and latitude, respectively). In these notations,

$$
M_{x}+i M_{y}=a_{1} e^{i\left(\phi_{1}+\chi_{1}\right)}+a_{3} e^{i\left(\phi_{3}+\chi_{3}\right)},
$$

where $a_{k} \equiv g_{k}^{n}\left|\Delta_{k}\right| \sin \theta_{k} /\left(g_{1}^{n}+g_{3}^{n}\right) \sqrt{h_{k}^{2}-\left|\Delta_{k}\right|^{2}}$, so that the $S$ phase and the longitude always come together.

The DOS is determined from $G_{2}$ and generally is different for the opposite spin directions,

$$
\nu^{\uparrow, \downarrow}(\varepsilon)=\nu_{0}^{\uparrow, \downarrow} \operatorname{Re}\left(1-\frac{a_{1}^{2}+a_{3}^{2}+2 a_{1} a_{3} \cos (\phi \pm \chi)}{\left(M_{0}-i \epsilon / E_{\mathrm{Th}}\right)^{2}}\right)^{-1 / 2},
$$

where $\nu_{0}^{\uparrow, \downarrow}$ is the normal-state DOS. As shown in Fig. 2(a), $\nu$ always exceeds the normal-state value at $\varepsilon \ll E_{\mathrm{Th}}$. This is in contrast with a suppression of $\nu$ manifesting the conventional proximity effect. Such an enhanced DOS is therefore a signature of TPE. The peak at small energies [Fig. 2(a)] is followed by a wide and shallow minimum at larger energies $\left(\epsilon \sim 3-4 E_{\mathrm{th}}\right)$, not shown in the figure. The DOS in the minimum is smaller than $\nu_{0}$ so that the total number of states remains unchanged. The dependence of $\nu$ on the $\mathrm{S}$ phase/longitude is also characteristic of TPE. As seen from Eq. (6), for a given spin direction the effect of $S$ phase can always be compensated by a rotation of one of the magnetization directions $\boldsymbol{n}_{1,3}$ about $\boldsymbol{n}_{2}$. While for a $\mathrm{F}$ metal the total DOS $\nu=\nu^{\dagger}+\nu^{\downarrow}$ is an even function of $\phi$ and $\chi$, [Fig. 2(b)], this is generally not so for a half-metal. In the latter case, for a symmetric setup $a_{1}=a_{3}$, the DOS
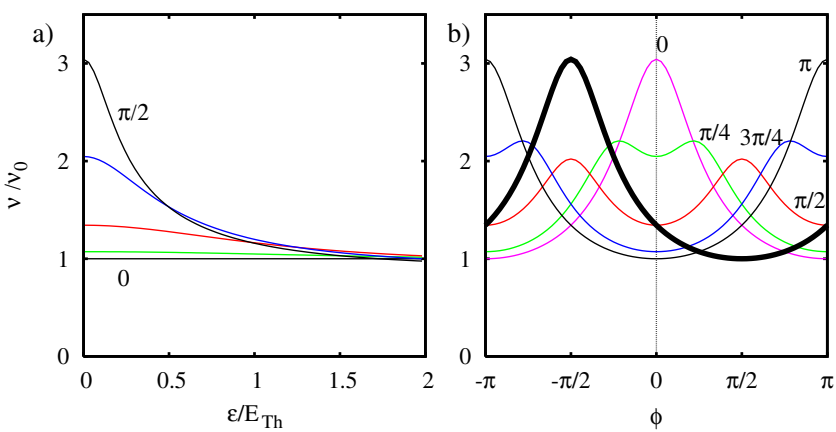

FIG. 2 (color online). The DOS in the central node (a) versus the energy for $\phi=0$ and $\chi=0$ where $\theta_{1}=\theta_{3}$ increases from 0 to $\pi / 2$ with a step $\pi / 8$. (b) The total DOS for a ferromagnetic metal at zero energy versus $\phi$ for $\theta_{1}=\theta_{3}=\pi / 2$ where $\chi$ increases from 0 to $\pi$ with a step $\pi / 4$. Upon increasing $\chi$ a single peak at $\phi=0$ splits in two. The thick curve corresponds to a half-metal with $\chi=\pi / 2$. For both graphs, $h_{1,3} / \Delta_{1,3}=$ $18 / 17$.

$\nu(0)$ can be modulated by either phase from 1 to its maximum value $1 / \sqrt{1-4 a_{1}^{2} / M_{0}^{2}}$.

In the model under consideration, the Josephson current is given by the Keldysh component of the matrix current through either junction $g_{1,3}^{n}$ integrated over energy,

$$
I=-\frac{g_{1}^{n}}{4 e} \int_{-\infty}^{\infty} d \epsilon \operatorname{Re} \operatorname{Tr} \tau_{3}\left[G_{1}, G_{2}\right] \tanh \frac{\epsilon}{2 T} .
$$

The block structure of $G_{2}$ gives rise to two contributions to the current that correspond to opposite spin directions. The integral in Eq. (7) is logarithmic, converging at energies $E_{\mathrm{Th}} \ll \epsilon \ll \Delta_{\text {cut }}, \quad \Delta_{\text {cut }} \simeq \min (\Delta, h, h-\Delta)$. We assume $T \ll E_{\mathrm{Th}}$. With logarithmic accuracy,

$$
I_{\uparrow, \downarrow}=-\frac{2 a_{1} a_{3}\left(g_{1}^{n}+g_{3}^{n}\right)}{e} \sin (\phi \pm \chi) E_{\mathrm{Th}} \ln \left(\frac{\Delta_{\mathrm{cut}}}{E_{\mathrm{Th}}}\right) .
$$

Both the scale of the current $\left(I \simeq g E_{\mathrm{Th}} / e\right)$ and the logarithmic structure are similar to the common proximity effect. The difference is the dependence of the current on the magnetization directions. For a $\mathrm{F}$ metal, the total current $I_{\uparrow}+I_{\downarrow}$ is odd in $\phi$. Interestingly, the sign of the Josephson current is opposite to that in a common Josephson contact provided $|\chi|<\pi / 2$. This signals a $\pi$ junction $[5,6,15]$, which can be changed to a common 0 junction by changing $\chi$. Since the accuracy of the logarithmic approximation is always questionable, we evaluate the integral numerically assuming $\Delta_{\text {cut }} / E_{\mathrm{Th}}=50$. The resulting dependence of the current on both phases is not precisely harmonic, though close to it (Figs. 3 and 4). As seen in Fig. 3(b), the second harmonic of the current becomes dominant in the vicinity of $\chi=\pi / 2$. This implies that the transition between " $\pi$ " and " 0 " states follows a scenario of Ref. [16] where both states are stable in this vicinity.

For a half-metal, the situation is very different. In this case, the $\mathrm{S}$ phase corresponding to the energy minimum 

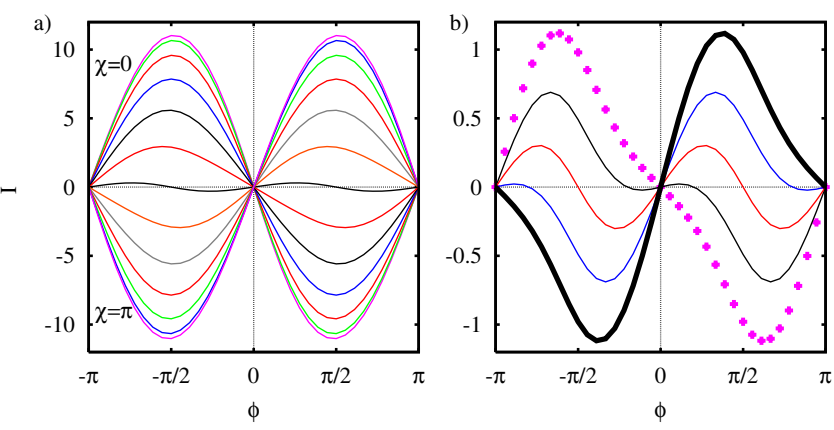

FIG. 3 (color online). The Josephson current for a ferromagnetic metal versus $\phi$ for different $\chi$ : (a) varying from 0 to $\pi$ with a step $\pi / 12$. The first harmonic dominates the current except the vicinity of $\chi=\pi / 2$ where it changes the sign. (b) The vicinity of the " $0-\pi$ " transition. Here $\chi$ varies from $\pi / 2-\pi / 36(\pi$ state, dotted line) through $\pi / 2-\pi / 72, \pi / 2, \pi / 2+\pi / 72$ (bistable, thin lines) to $\pi / 2+\pi / 36$ (0 state, thick line).

just follows $\chi$ and changes continuously instead of "jumping" between the values of 0 and $\pi$. There is a finite supercurrent at zero phase difference. Therefore, rotation of the magnetizations $\boldsymbol{n}_{1,3}$ about $\boldsymbol{n}_{2}$ is equivalent to the effect of extra magnetic flux $\chi \Phi_{0} / 2 \pi$ enclosed in a large loop that includes the junction. In our opinion, this facilitates an unambiguous experimental verification of TPE. In both cases, not only is the supercurrent zero at the energetically favorable phase difference, but also TPE is reduced, vanishing completely for an ideally symmetric setup. This is because TPE increases the electron energy in the central node, as is seen from the fact that the DOS is enhanced at low energies.

In conclusion, we have proposed a simple model for fully developed triplet proximity effect in S/F/S structures. In contrast to the common proximity effect, TPE enhances the DOS at low energies [17]. The Josephson current exhibits a peculiar dependence on the magnetization configuration that is essentially different for a $\mathrm{F}$ metal and a half-metal. These are signatures of TPE to be observed experimentally. It is important to stress that our conclu-

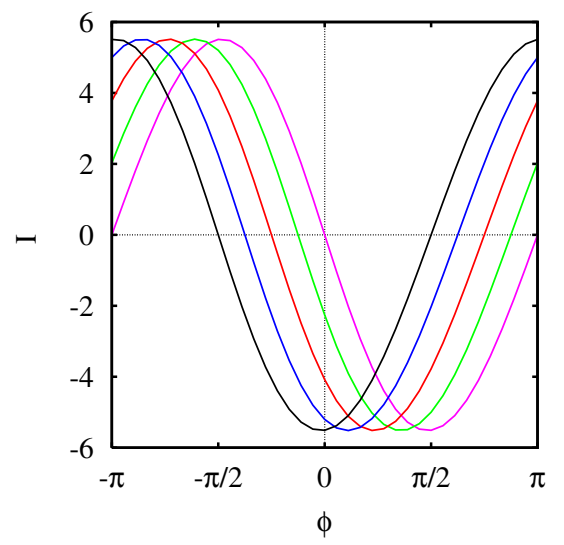

FIG. 4 (color online). The Josephson current for a half-metal versus $\phi$ for $\chi$ varying from 0 to $\pi / 2$ with a step $\pi / 8$. The $I-$ $\phi$ curves shift to the left with increasing $\chi$. sions, by virtue of the circuit theory, pertain to sufficiently disordered ("dirty") structures only. The TPE in the clean limit has been addressed in Ref. [18], where the DOS was found to exhibit a complicated pattern of suppression and enhancement. The enhancement of local DOS was also predicted in several clean systems [19]. Experimentally, structures with lateral design are believed to be dirty.

We appreciate useful discussions with Ya. M. Blanter, G.E.W. Bauer, T. M. Klapwijk, R. S. Keizer, and E. B. Sonin, and valuable communications with Y. Asano and A.F. Volkov. The work was supported by EC NMP2CT2003-505587 "SFINX."

[1] A. I. Larkin and Y. N. Ovchinnikov, Sov. Phys. JETP 20, 762 (1965); P. Fulde and R. A. Ferrell, Phys. Rev. 135, A550 (1964).

[2] For a review, see F. S. Bergeret, A. F. Volkov, and K. B. Efetov, Rev. Mod. Phys. 77, 1321 (2005).

[3] M. A. M. Gijs and G.E. W. Bauer, Adv. Phys. 46, 285 (1997); I. Žutić, J. Fabian, and S. Das Sarma, Rev. Mod. Phys. 76, 323 (2004).

[4] P. M. Tedrow and R. Meservey, Phys. Rev. Lett. 26, 192 (1971); G. Deutscher and F. Meunier, Phys. Rev. Lett. 22, 395 (1969).

[5] V. V. Ryazanov et al., Phys. Rev. Lett. 86, 2427 (2001).

[6] A. F. Volkov, F. S. Bergeret, and K. B. Efetov, Phys. Rev. Lett. 90, 117006 (2003); F. S. Bergeret, A. F. Volkov, and K. B. Efetov, Phys. Rev. B 68, 064513 (2003); A. F. Volkov, Ya. V. Fominov, and K. B. Efetov, Phys. Rev. B 72, 184504 (2005).

[7] R. S. Keizer et al., Nature (London) 439, 825 (2006).

[8] A. I. Buzdin, A. V. Vedyayev, and N. V. Ryzhanova, Europhys. Lett. 48, 686 (1999).

[9] D. Huertas-Hernando, Yu. V. Nazarov, and W. Belzig, Phys. Rev. Lett. 88, 047003 (2002).

[10] V. T. Petrashov et al., Phys. Rev. Lett. 83, 3281 (1999); M. Giroud et al., Phys. Rev. B 58, R11872 (1998).

[11] W. Belzig et al., Phys. Rev. B 62, 9726 (2000).

[12] Yu. V. Nazarov, Phys. Rev. Lett. 73, 1420 (1994); Superlattices Microstruct. 25, 1221 (1999).

[13] A. I. Larkin and Yu. N. Ovchinnikov, Sov. Phys. JETP 28, 1200 (1969).

[14] Recent numerical results (Y. Asano, Y. Tanaka, and A. Golubov, cond-mat/0609566) suggest that the interface between a half-metal and a superconductor may play a role of such a reservoir.

[15] D. J. van Harlingen, Rev. Mod. Phys. 67, 515 (1995); J. J. A. Baselmans et al., Phys. Rev. Lett. 89, 207002 (2002); J. A. van Dam et al., Nature (London) 442, 667 (2006).

[16] N. M. Chtchelkatchev et al., JETP Lett. 74, 323 (2001).

[17] It is shown in a recent paper (T. Yokoyama, Y. Tanaka, and A. A. Golubov, cond-mat/0610608) that the DOS at Fermi energy is increased if the proximity effect is due to oddfrequency pairing, as it is for TPE.

[18] M. Eschrig et al., Phys. Rev. Lett. 90, 137003 (2003).

[19] M. Krawiec, B. L. Györffy, and J. F. Annett, Phys. Rev. B 70, 134519 (2004); K. Kuboki, J. Phys. Soc. Jpn. 68, 3150 (1999). 